\title{
COVID-19 vaccine acceptance, barriers and facilitators among healthcare workers in Pakistan
}

\author{
Khalid Rehman ( $\nabla$ drkhalid.iph@kmu.edu.pk) \\ Khyber Medical University \\ Muhammad Hakim \\ Khyber Medical University \\ Nauman Arif \\ Khyber Medical University \\ Siraj UI Islam \\ Lady Reading Hospital \\ Abdul Saboor \\ Khyber Medical University \\ Muhammad Asif \\ Khyber Medical University \\ Michael Ramharter \\ Bernhard Nocht Institute for Tropical Medicine
}

\section{Research Article}

Keywords: COVID-19, vaccine acceptance, Health care workers, Khyber Pakhtunkhwa, Pakistan

Posted Date: April 26th, 2021

DOI: https://doi.org/10.21203/rs.3.rs-431543/v2

License: (c) (1) This work is licensed under a Creative Commons Attribution 4.0 International License. Read Full License 


\section{Abstract}

\section{Background}

Vaccines are one of the main cornerstones in the global initiatives to control the COVID-19 pandemic. National vaccination programs largely depend on vaccine availability and acceptance by a large proportion of the population to confer a measurable impact on SARS-CoV-2 transmission. In this study we determine the acceptance, barriers and facilitators of COVID-19 vaccination amongst healthcare workers (HCWs) in Pakistan

\section{Methods}

A structured and validated questionnaire was used in an online cross-sectional study. Health care workers residing in Pakistan were invited between $31^{\text {st }}$ January to $9^{\text {th }}$ February to participate in this survey

\section{Results}

A total of 436 health-care workers took part in this survey and among those 327 (75\%) were doctors. $260(60 \%)$ health-care workers indicated that they would accept vaccination against COVID-19. 308 (71\%) were completely confident or confident in using USA, UK manufactured COVID-19 vaccine. The acceptance concerning a potential Chinese vaccine was 267 (61\%). 292 (67\%) strongly agreed or agreed that they are concerned about the efficacy of COVID-19 vaccination while the concerns for safety were $293(67 \%)$

\section{Conclusions}

About a third of HCWs, a group generally biased towards early acceptance of health interventions - expressed concerns or refusal of vaccination against COVID-19 in this survey. A dedicated communication plan and information campaign addressing the issues of efficacy, quality, logistics and religious concerns is needed to address these hurdles to allow for a successful national vaccination campaign.

\section{Background}

The recent pandemic of COVID-19 caused by severe acute respiratory syndrome coronavirus 2 (SARS-CoV-2) is a major threat globally and particularly to Southeast Asian countries $(1,2)$. The pandemic demands for intervention on many fronts, from prevention to testing to treatment. Vaccines are among the most effective public health interventions in preventing morbidity, mortality and public health costs caused by infectious diseases (3) and are therefore a cornerstone in the control measures of the global COVID-19 pandemic.

Since the very beginning of COVID-19 pandemic, researchers around the globe have been working tirelessly on countermeasures to contain the pandemic. While it was not yet possible to identify particular highly efficacious antiviral drugs, there was significant progress in the development of a vaccine to fight the disease (4). Several different vaccines, all with promising efficacy and protection, are produced at a record pace of less than a year after the pandemic begins (5).Arguably, vaccine hesitancy and rejection by a majority of the population are among the prime concerns for a successful vaccination campaign aiming at achieving herd immunity (6). Studies from different countries including affluent nations have shown discouraging results on the effectiveness of vaccination programs (7). It is therefore of high importance to identify potential barriers to vaccine uptake early on in the fight against COVID-19.

Pakistan has a history of problems with vaccine uptake and acceptability (8-10). Low coverage rates of regular immunization programs and the large number of individuals who fail to vaccinate their children in polio immunization campaigns, despite stringent by-laws and vigorous anti-polio drives, highlight this reality.

Healthcare workers (HCWs) are particularly exposed to and a main source of nosocomial transmission of COVID-19, which makes them an important target group for vaccination (11). It is based on this understanding that HCW will constitute the 
priority group for the national COVID-19 vaccination program in Pakistan. This program is conceived as a multi-phase program due to shortage of available vaccines. Vaccine uptake and addressing of concerns observed in the first vaccinated groups will prove crucial for the further success of the vaccination program as HCW may act as main influencers of the general public in adapting any healthy behavior. This study therefore set out to investigate the acceptance, perceptions and concerns of HCW in Pakistan about the imminent national COVID-19 vaccine program.

\section{Methods}

A cross-sectional study was conducted inviting healthcare workers in Pakistan during January 2021 via online social media platforms. The target population was healthcare workers aged $\geq 21$ years with a valid registration in Pakistan with their respective regulatory body. The survey was advertised via three social media platforms (Facebook, WhatsApp and Twitter). Data was collected using self-reported, structured questionnaire adapted from Lin Y et all (12). Data were collected via an online Google forms. The survey was open for response between $31^{\text {st }}$ January to $9^{\text {th }}$ February. The identity of health care personnel validated by their registration number, which was kept confidential.

Data analysis:

Data were extracted from the online Google forms, transferred and analyzed by using STATA Version 14 . All the categorical variables (gender, professional role, vaccine acceptance etc) were described as frequencies and percentages. Group-testing was performed using chi square test with $p \leq 0.05$ considered significance.

\section{Results}

A total of 436 responses were received in the two weeks period. Of these, 327 (75\%) were medical doctors, 34 (7.80\%) were nurses, 39 (8.94\%) were paramedical staff and 36 (8.26\%) were other health staff (Table 1).

The respondents belong to all provinces and regions of Pakistan but the majority participated from the province Khyber Pakhtunkhwa (KPK) 335 (77\%). 47 (11\%), 27 (6\%), 12 (3\%), 11 (3\%) were from Punjab, Sindh, Federal and Baluchistan respectively. Two third of the respondents were in the age category 19-39 years, $n=293(67 \%)$. The most frequently reported category for years of experience was 0 - 5 years reported by, $n=176(40 \%)$. Most of them $n=180,(41 \%)$ worked in tertiary care hospitals while $n=99(23 \%), n=44(10 \%), n=44(10 \%)$ worked in secondary care hospital, primary care hospitals and emergency operation centers respectively. Respondent's further details on the characteristics are provided in Table 1.

Out of 436 respondents, $n=133$ (31\%) had tested COVID-19 positive. Total 68 (16\%) respondents have an existing chronic disease such as cardiovascular disease, diabetes and chronic respiratory tract disease. When asked, about if they had adequate information regarding the COVID-19 vaccine, only 202 (46\%) of the health-care workers agreed with having adequate information.

$260(60 \%)$ health-care workers responded that they would accept receiving vaccine against COVID-19 if available, 136 (31\%) would wait \& see and $16(4 \%)$ would categorically refuse vaccination. $308(71 \%)$ were completely confident or confident in using USA, UK manufactured COVID-19 vaccine, whereas 267 (61\%) were completely confident or confident in using China manufactured COVID-19 vaccine.

326 (75\%) strongly agreed or agreed that they will feel less worried about getting COVID-19 if vaccinated. 329 (75\%) acknowledged that vaccination decreases their risk of contracting COVID-19 and its complications. 174 (40\%) indicated that they are worried about possible side effects of COVID-19 vaccination.

Total 292 (67\%) strongly agreed or agreed that they are concerned about the efficacy of COVID-19 vaccination whereas 293 (67\%) are concerned about the safety of COVID-19 vaccination including 238 (54.5\%) voicing concerns about potential fake and faulty COVID-19 vaccine. 
$320(73.3 \%)$ indicated concerns about the cold chain maintenance of COVID-19 vaccine and 125 (28.6\%) about the permissibility of COVID-19 vaccine on religious ground. 128 participants $(29.3 \%$, ) assumed that there might be hidden motives behind eagerness of authorities to administer / provide COVID-19 vaccine free. Respondent's further details on the respondent's characteristics are provided in Table 02(a-c).

The majority of respondents were from the KPK province of Pakistan. Further details of the KPK respondent's characteristics are provided in Table 03(a-c)

A separate analysis was run for total 133 respondents who were COVID-19 positive among which a majority were doctors and working at tertiary care hospitals $67(50 \%)$ of Pakistan.

72 (54\%) responded that they are willing to take a COVID-19 vaccine if available. 95 (71\%) strongly agreed or agreed that they would feel less worried of contracting COVID-19 if vaccinated. 31 (23\%) were concerned about permissibility of COVID-19 vaccine on religious grounds. The details given in Table 04.

\section{Discussion}

Knowledge, attitude and practices of HCWs is important to understand to guide vaccination programs. Pakistan is a particularly complex region for population based vaccination programs due to a history of vaccine hesitancy in the past. This survey therefore aimed to address proactively the major barriers for vaccine acceptance to help guide information management to effectively accompany the national COVID-19 response.

Importantly, about one third of health care workers in our survey indicated to postpone or refuse taking a COVID-19 vaccine. Given that these individuals are HCW, disproportionally young and well educated this seems a rather high proportion. This group of respondents is thought to be rather geared towards acceptance of evidence based interventions, which makes the significant proportion of hesitant individuals even more important. In similar studies done in health workers of other countries vaccine acceptance has been reported to be within a borad range from $28 \%$ in DRC, $40 \%$ in Hong Kong, $61 \%$ in Israel, $63 \%$ Hong Kong, up to $77 \%$ in France (6,13-16). Interestingly, HCWs showed a trend towards more trust in US/UK manufactured vaccines than on a Chinese vaccine. This is important for the Pakistani context as two Chinese vaccines (Sinopharm, CanSino) are planned to be the main vaccines for the national control program. Concerns regarding logistics and quality assurance are justified concerns that need to be addressed both on the operation level as well as should be included in information campaigns. Importantly, one third of respondents also indicated issues with religious permissibility as a concern. This particular topic requires further investigation and engagement with religious leaders to potentially overcome this important hurdle in the effective implementation of the national COVID-19 vaccination program.

As mentioned above, this survey is probably not fully representative for the Pakistani population as it significantly overrepresents young male HCWs with high education from one part of Pakistan. Nevertheless, it is important information from a country with history of vaccine hesitancy and a region with multiple health issues to tackle.

\section{Conclusion}

In summary, this survey indicates the overall acceptance of an anti-COVID-19 vaccination program and highlights important aspects to be included in engagement with national stakeholders and to be communicated in national information campaigns. Religious beliefs also need to be taken into account while planning a communication strategy.

\section{List Of Abbreviations}

HCW Health care workers

KPK Khyber Pakhtunkhwa 


\section{Declarations}

Ethical approval

Ethical approval has been taken from the ethics committee of Khyber Medical University.

Consent

Consent from participants was taken, once they agreed only then the survey went further.

\section{Availability of data}

All the collected data is available with the corresponding author.

\section{Competing interests}

The authors declare that they have no competing interests.

\section{Funding}

No funding was received for this research.

\section{Authors contributions}

KR, conceived the idea, contributed to the design, proposal development, writing of the manuscript and approved the submitted version of the manuscript.

MH, helped in proposal development, data analysis, writing of manuscript and approved the submitted version of the manuscript.

NA, analyzed, interpreted the data and approved the submitted version of the manuscript.

SI, helped in data collection, writing of manuscript and approved the submitted version of the manuscript.

AS, helped in writing the proposal, data collection, ethical approval and approved the submitted version of the manuscript.

MA, helped in proposal development, finalizing the data collection tool and approved the submitted version of the manuscript.

MR, helped data analysis, interpretation, substantively revised manuscript and approved the submitted version of the manuscript.

\section{Acknowledgements}

Dr. Zohaib Khan.

Director ORIC. Khyber Medical University. Peshawar. Pakistan.

Dr. Zeeshan Kibria.

Deputy Director ORIC. Peshawar. Pakistan. Khyber Medical University.

\section{Authors information}

KR has a PhD in Public Health from Medical University of Vienna, Austria.

He is working as assistant professor of public health in Khyber Medical University, Peshawar. 


\section{References}

1. Bhutta ZA, Basnyat B, Saha S, Laxminarayan R. Covid-19 risks and response in South Asia. British Medical Journal Publishing Group; 2020.

2. Lim W, Liang C, Assantachai P, Auyeung TW, Kang L, Lee W, et al. COVID-19 and older people in Asia: Asian Working Group for Sarcopenia calls to action. Geriatr Gerontol Int. 2020;20(6):547-58.

3. (CDC C for DC and P. Ten great public health achievements-United States, 1900-1999. MMWR Morb Mortal Wkly Rep. 1999;48(12):241-3.

4. Dutta AK. Vaccine Against Covid-19 Disease-Present Status of Development. Indian J Pediatr. 2020;1-7.

5. Walsh EE, Frenck Jr RW, Falsey AR, Kitchin N, Absalon J, Gurtman A, et al. Safety and immunogenicity of two RNA-based Covid-19 vaccine candidates. N Engl J Med. 2020;383(25):2439-50.

6. Dror AA, Eisenbach N, Taiber S, Morozov NG, Mizrachi M, Zigron A, et al. Vaccine hesitancy: the next challenge in the fight against COVID-19. Eur J Epidemiol. 2020;35(8):775-9.

7. Grech V, Gauci C, Agius S. Vaccine hesitancy among Maltese healthcare workers toward influenza and novel COVID-19 vaccination. Early Hum Dev. 2020;105213.

8. Khattak FA, Rehman K, Shahzad M, Arif N, Ullah N, Kibria Z, et al. Prevalence of Parental refusal rate and its associated factors in routine immunization by using WHO Vaccine Hesitancy tool: A Cross sectional study at district Bannu, KP, Pakistan. Int J Infect Dis. 2021;104:117-24.

9. Murakami H, Kobayashi M, Hachiya M, Khan ZS, Hassan SQ, Sakurada S. Refusal of oral polio vaccine in northwestern Pakistan: a qualitative and quantitative study. Vaccine. 2014;32(12):1382-7.

10. Jamal D, Zaidi S, Husain S, Orr DW, Riaz A, Farrukhi AA, et al. Low vaccination in rural Sindh, Pakistan: A case of refusal, ignorance or access? Vaccine. 2020;38(30):4747-54.

11. Haviari S, Bénet T, Saadatian-Elahi M, André P, Loulergue P, Vanhems P. Vaccination of healthcare workers: a review. Hum Vaccin Immunother. 2015;11(11):2522-37.

12. Lin $Y$, Hu Z, Zhao Q, Alias H, Danaee M, Wong LP. Understanding COVID-19 vaccine demand and hesitancy: A nationwide online survey in China. PLoS Negl Trop Dis. 2020;14(12):e0008961.

13. Kabamba Nzaji M, Kabamba Ngombe L, Ngoie Mwamba G, Banza Ndala DB, Mbidi Miema J, Luhata Lungoyo C, et al. Acceptability of Vaccination Against COVID-19 Among Healthcare Workers in the Democratic Republic of the Congo. Pragmatic Obs Res [Internet]. 2020 Oct 29;11:103-9. Available from: https://pubmed.ncbi.nlm.nih.gov/33154695

14. Wang K, Wong ELY, Ho KF, Cheung AWL, Chan EYY, Yeoh EK, et al. Intention of nurses to accept coronavirus disease 2019 vaccination and change of intention to accept seasonal influenza vaccination during the coronavirus disease 2019 pandemic: A cross-sectional survey. Vaccine [Internet]. 2020;38(45):7049-56. Available from:

https://www.sciencedirect.com/science/article/pii/S0264410X20311750

15. Kwok KO, Li K-K, WEI WI, Tang A, Wong SYS, Lee SS. Influenza vaccine uptake, COVID-19 vaccination intention and vaccine hesitancy among nurses: A survey. Int J Nurs Stud [Internet]. 2021;114:103854. Available from:

https://www.sciencedirect.com/science/article/pii/S002074892030345X

16. Gagneux-Brunon A, Detoc M, Bruel S, Tardy B, Rozaire O, Frappe P, et al. Intention to get vaccinations against COVID-19 in French healthcare workers during the first pandemic wave: a cross-sectional survey. J Hosp Infect [Internet]. 2021;108:168-73. Available from: https://www.sciencedirect.com/science/article/pii/S0195670120305442

\section{Tables}




\begin{tabular}{|c|c|c|c|c|c|c|c|}
\hline \multirow[t]{2}{*}{ VARIABLE } & \multirow[t]{2}{*}{ CATEGORIES } & \multicolumn{6}{|c|}{ HEALTH CARE GROUPS } \\
\hline & & $\begin{array}{l}\text { DOCTORS } \\
\text { N=327 } \\
\text { (75.00\%) }\end{array}$ & $\begin{array}{l}\text { NURSES } \\
\mathrm{N}=34 \\
(7.80 \%)\end{array}$ & $\begin{array}{l}\text { PARAMEDICS } \\
\mathrm{N}=39 \\
(8.94 \%)\end{array}$ & $\begin{array}{l}\text { OTHERS } \\
\mathrm{N}=36 \\
(8.26 \%)\end{array}$ & $\begin{array}{l}\text { Total } \\
N=436 \\
(100 \%)\end{array}$ & P-value \\
\hline \multirow{7}{*}{ Location } & $\begin{array}{l}\text { Khyber } \\
\text { Pakhtunkhwa }\end{array}$ & $242(74.01)$ & $29(85.29)$ & $37(94.87)$ & $27(75.00)$ & $335(76.83)$ & \multirow{7}{*}{0.70} \\
\hline & Punjab & $38(11.62)$ & $3(8.82)$ & $0(0.00)$ & $6(16.67)$ & $47(10.78)$ & \\
\hline & Sindh & $23(7.03)$ & $2(5.88)$ & $1(2.56)$ & $1(2.78)$ & $27(6.19)$ & \\
\hline & Baluchistan & $10(3.06)$ & $0(0.00)$ & $0(0.00)$ & $1(2.78)$ & $11(2.52)$ & \\
\hline & $\begin{array}{l}\text { Azad Jammu \& } \\
\text { Kashmir }\end{array}$ & $3(0.92)$ & $0(0.00)$ & $0(0.00)$ & $0(0.00)$ & $3(0.69)$ & \\
\hline & Federal & $10(3.06)$ & $0(0.00)$ & $1(2.56)$ & $1(2.78)$ & $12(2.75)$ & \\
\hline & Gilgit Baltistan & $1(0.31)$ & $0(0.00)$ & $0(0.00)$ & $0(0.00)$ & $1(0.23)$ & \\
\hline \multirow[t]{2}{*}{ Gender } & Female & $72(22.02)$ & $19(55.88)$ & $5(12.82)$ & $5(13.89)$ & $101(23.17)$ & \multirow[t]{2}{*}{$<0.001$} \\
\hline & Male & $255(77.98)$ & $15(44.12)$ & $34(87.18)$ & $31(86.11)$ & $335(76.83)$ & \\
\hline \multirow{3}{*}{$\begin{array}{l}\text { Category of } \\
\text { age }\end{array}$} & $19-39$ & $200(61.16)$ & $32(94.12)$ & $30(76.92)$ & $31(86.11)$ & $293(67.20)$ & \multirow[t]{3}{*}{$<0.001$} \\
\hline & $40-60$ & $116(35.47)$ & $2(5.88)$ & $8(20.51)$ & $5(13.89)$ & $131(30.05)$ & \\
\hline & $61-81$ & $11(3.36)$ & $0(0.00)$ & $1(2.56)$ & $0(0.00)$ & $12(2.75)$ & \\
\hline \multirow{3}{*}{$\begin{array}{l}\text { Category of } \\
\text { work } \\
\text { experience in } \\
\text { years }\end{array}$} & $0-5$ Years & $117(35.78)$ & $21(61.76)$ & $22(56.41)$ & $16(44.44)$ & $176(40.37)$ & \multirow[t]{3}{*}{$<0.001$} \\
\hline & 6-10 Years & $76(23.24)$ & $10(29.41)$ & $5(12.82)$ & 14(38.89) & $105(24.08)$ & \\
\hline & Above 10 Years & $134(40.98)$ & $3(8.82)$ & $12(30.77)$ & $6(16.67)$ & 155(35.55) & \\
\hline \multirow{6}{*}{$\begin{array}{l}\text { Working at } \\
\text { which health } \\
\text { care level? }\end{array}$} & $\begin{array}{l}\text { COVID-19 } \\
\text { Facility/Hospitals }\end{array}$ & $3(0.92)$ & $0(0.00)$ & $6(15.38)$ & $1(2.78)$ & $10(2.29)$ & \multirow[t]{6}{*}{$<0.001$} \\
\hline & $\begin{array}{l}\text { Emergency } \\
\text { Operation } \\
\text { Center(National, } \\
\text { provincials and } \\
\text { districts) }\end{array}$ & $50(15.29)$ & $0(0.00)$ & $1(2.56)$ & $3(8.33)$ & $54(12.39)$ & \\
\hline & $\begin{array}{l}\text { Primary Health } \\
\text { Care Facility }\end{array}$ & $34(10.40)$ & $0(0.00)$ & $9(23.08)$ & $1(2.78)$ & $44(10.09)$ & \\
\hline & $\begin{array}{l}\text { Secondary Health } \\
\text { Care Facility }\end{array}$ & $71(21.71)$ & $14(41.18)$ & $7(17.95)$ & $7(19.44)$ & $99(22.71)$ & \\
\hline & $\begin{array}{l}\text { Tertiary Health } \\
\text { Care Facility }\end{array}$ & $143(43.73)$ & $18(52.94)$ & $10(25.64)$ & $9(25.00)$ & $180(41.28)$ & \\
\hline & Other & $26(7.95)$ & $2(5.88)$ & $6(15.38)$ & $15(41.67)$ & $49(11.24)$ & \\
\hline \multirow{3}{*}{$\begin{array}{l}\text { Are you } \\
\text { working at } \\
\text { facility/ward } \\
\text { dedicated for } \\
\text { COVID-19 } \\
\text { patients? }\end{array}$} & No & $153(46.79)$ & $11(32.35)$ & $17(43.59)$ & $20(55.56)$ & $201(46.10)$ & \multirow[t]{3}{*}{0.01} \\
\hline & Yes & 109 (33.33) & $16(47.06)$ & $20(51.28)$ & $6(16.67)$ & $151(34.63)$ & \\
\hline & Both & $65(19.88)$ & $7(20.59)$ & $2(5.13)$ & $10(27.78)$ & $84(19.27)$ & \\
\hline
\end{tabular}


02 c: COVID-19 vaccine acceptance, barriers and facilitators amongst health care workers of Pakistan

\begin{tabular}{|c|c|c|c|c|c|c|c|}
\hline \multirow[t]{2}{*}{ BLES } & \multirow[t]{2}{*}{ CATEGORIES } & \multicolumn{6}{|c|}{ HEALTH CARE GROUPS } \\
\hline & & $\begin{array}{l}\text { DOCTORS } \\
N=327 \\
(75.00 \%)\end{array}$ & $\begin{array}{l}\text { NURSES } \\
\text { N=34 } \\
(7.80 \%)\end{array}$ & $\begin{array}{l}\text { PARAMEDICS } \\
\mathrm{N}=39 \\
(8.94 \%)\end{array}$ & $\begin{array}{l}\text { OTHERS } \\
N=36 \\
(8.26 \%)\end{array}$ & $\begin{array}{l}\text { Total } \\
N=436 \\
(100 \%)\end{array}$ & P-value \\
\hline \multirow{5}{*}{$\begin{array}{l}\text { oncerned about } \\
\text { Cold Chain } \\
\text { nance } \\
\text {-19 vaccine? }\end{array}$} & Agree & $148(45.26)$ & $17(50.00)$ & $15(38.46)$ & $14(38.89)$ & $194(44.50)$ & \multirow[t]{5}{*}{0.12} \\
\hline & Disagree & $21(6.42)$ & $2(5.88)$ & $2(5.13)$ & $2(5.56)$ & $27(6.19)$ & \\
\hline & Neutral & $58(17.74)$ & $9(26.47)$ & $10(25.64)$ & $9(25.00)$ & $86(19.72)$ & \\
\hline & $\begin{array}{l}\text { Strongly } \\
\text { agree }\end{array}$ & $99(30.28)$ & $6(17.65)$ & $10(25.64)$ & $11(30.56)$ & $126(28.90)$ & \\
\hline & $\begin{array}{l}\text { Strongly } \\
\text { disagree }\end{array}$ & $1(0.31)$ & $0(0.00)$ & $2(5.13)$ & $0(0.00)$ & $3(0.69)$ & \\
\hline \multirow{5}{*}{$\begin{array}{l}\text { oncerned about } \\
\text { ssibility of } \\
\text { )-19 vaccine on } \\
\text { us grounds? }\end{array}$} & Agree & $56(17.13)$ & $12(35.29)$ & $10(25.64)$ & $10(27.78)$ & $88(20.18)$ & \multirow[t]{5}{*}{0.005} \\
\hline & Disagree & $121(37.00)$ & $9(26.47)$ & $7(17.95)$ & $11(30.56)$ & $148(33.94)$ & \\
\hline & Neutral & $83(25.38)$ & $12(35.29)$ & $12(30.77)$ & $8(22.22)$ & 115(26.38) & \\
\hline & $\begin{array}{l}\text { Strongly } \\
\text { agree }\end{array}$ & $24(7.34)$ & $1(2.94)$ & $8(20.51)$ & $4(11.11)$ & $37(8.49)$ & \\
\hline & $\begin{array}{l}\text { Strongly } \\
\text { disagree }\end{array}$ & $43(13.15)$ & $0(0.00)$ & $2(5.13)$ & $3(8.33)$ & $48(11.01)$ & \\
\hline \multirow{5}{*}{$\begin{array}{l}\text { might be } \\
1 \text { motives } \\
1 \text { eagerness of } \\
\text { ities to } \\
\text { ister/provide } \\
\text { e free for all } \\
\text { workers? }\end{array}$} & Agree & $47(14.37)$ & $16(47.06)$ & $9(23.08)$ & $11(30.56)$ & $83(19.04)$ & \multirow[t]{5}{*}{$<0.001$} \\
\hline & Disagree & $111(33.94)$ & $4(11.76)$ & $9(23.08)$ & $8(22.22)$ & $132(30.28)$ & \\
\hline & Neutral & $83(25.38)$ & $8(23.53)$ & $10(25.64)$ & $7(19.44)$ & $108(24.77)$ & \\
\hline & $\begin{array}{l}\text { Strongly } \\
\text { agree }\end{array}$ & $26(7.95)$ & $6(17.65)$ & $8(20.51)$ & $5(13.89)$ & $45(10.32)$ & \\
\hline & $\begin{array}{l}\text { Strongly } \\
\text { disagree }\end{array}$ & $60(18.35)$ & $0(0.00)$ & $3(7.69)$ & $5(13.89)$ & $68(15.60)$ & \\
\hline
\end{tabular}


2 a: COVID-19 Vaccine acceptance, barriers and facilitators amongst health care workers of Pakistan

\begin{tabular}{|c|c|c|c|c|c|c|c|}
\hline \multirow[t]{2}{*}{ iLES } & \multirow[t]{2}{*}{ CATEGORIES } & \multicolumn{6}{|c|}{ HEALTH CARE GROUPS } \\
\hline & & $\begin{array}{l}\text { DOCTORS } \\
N=327 \\
(75.00 \%)\end{array}$ & $\begin{array}{l}\text { NURSES } \\
\mathrm{N}=34 \\
(7.80 \%)\end{array}$ & $\begin{array}{l}\text { PARAMEDICS } \\
\mathrm{N}=39 \\
(8.94 \%)\end{array}$ & $\begin{array}{l}\text { OTHERS } \\
\mathrm{N}=36 \\
(8.26 \%)\end{array}$ & $\begin{array}{l}\text { Total } \\
N=436 \\
(100 \%)\end{array}$ & P-value \\
\hline \multirow{2}{*}{$\begin{array}{l}\text { iu ever tested } \\
\text { for } \\
19 \text { ? }\end{array}$} & No & $223(68.20)$ & $22(64.71)$ & $30(76.92)$ & $28(77.78)$ & $303(69.50)$ & \multirow[t]{2}{*}{0.42} \\
\hline & Yes & 104(31.80) & $12(35.29)$ & $9(23.08)$ & $8(22.22)$ & $133(30.50)$ & \\
\hline \multirow{2}{*}{$\begin{array}{l}\text { have an } \\
\text { I chronic } \\
\text { such as } \\
\text { ascular } \\
\text {, diabetes, } \\
\text { : Respiratory } \\
\text { sease? }\end{array}$} & No & $268(81.96)$ & $32(94.12)$ & $36(92.31)$ & $32(88.89)$ & $368(84.40)$ & \multirow[t]{2}{*}{0.09} \\
\hline & Yes & $59(18.04)$ & $2(5.88)$ & $3(7.69)$ & $4(11.11)$ & $68(15.60)$ & \\
\hline \multirow{3}{*}{$\begin{array}{l}\text { ieve you have } \\
\text { te information } \\
\text { ng COVID-19 } \\
\text { s? }\end{array}$} & I am not sure & $103(31.50)$ & $14(41.18)$ & $15(38.46)$ & $11(30.56)$ & $143(32.80)$ & \multirow[t]{3}{*}{0.31} \\
\hline & No & $71(21.71)$ & $9(26.47)$ & $3(7.69)$ & $8(22.22)$ & $91(20.87)$ & \\
\hline & Yes & $153(46.79)$ & $11(32.35)$ & $21(53.85)$ & $17(47.22)$ & $202(46.33)$ & \\
\hline \multirow{4}{*}{$\begin{array}{l}\text { nes against } \\
19 \text { infection } \\
\text { ailable would } \\
\text { e it? }\end{array}$} & I am not sure & $17(5.20)$ & $3(8.82)$ & $2(5.13)$ & $2(5.56)$ & $24(5.50)$ & \multirow[t]{4}{*}{0.24} \\
\hline & $\begin{array}{l}\text { I will wait } \\
\text { and see }\end{array}$ & $96(29.36)$ & $14(41.18)$ & $12(30.77)$ & $14(38.89)$ & $136(31.19)$ & \\
\hline & No & $10(3.06)$ & $1(2.94)$ & $1(2.56)$ & $4(11.11)$ & $16(3.67)$ & \\
\hline & Yes & $204(62.39)$ & $16(47.06)$ & $24(61.54)$ & $16(44.44)$ & $260(59.63)$ & \\
\hline \multirow{4}{*}{$\begin{array}{l}\text { iur confidence } \\
\text { IK,USA } \\
\text { ctured } \\
\text { ed) } \\
19 \text { vaccines? }\end{array}$} & $\begin{array}{l}\text { Completely } \\
\text { confident }\end{array}$ & 64(19.57) & $6(17.65)$ & $12(30.77)$ & $12(33.33)$ & $94(21.56)$ & \multirow[t]{4}{*}{$<0.001$} \\
\hline & $\begin{array}{l}\text { Completely } \\
\text { not confident }\end{array}$ & $23(7.03)$ & $11(32.35)$ & $4(10.26)$ & $5(13.89)$ & $43(9.86)$ & \\
\hline & Confident & 178(54.43) & $10(29.41)$ & $15(38.46)$ & $11(30.56)$ & $214(49.08)$ & \\
\hline & $\begin{array}{l}\text { Not } \\
\text { confident }\end{array}$ & $62(18.96)$ & $7(20.59)$ & $8(20.51)$ & $8(22.22)$ & $85(19.50)$ & \\
\hline \multirow{4}{*}{$\begin{array}{l}\text { ur confidence } \\
\text { 'hina } \\
\text { ctured } \\
19 \text { vaccine? }\end{array}$} & $\begin{array}{l}\text { Completely } \\
\text { confident }\end{array}$ & $50(15.29)$ & $3(8.82)$ & $16(41.03)$ & $11(30.56)$ & $80(18.35)$ & \multirow[t]{4}{*}{$<0.001$} \\
\hline & $\begin{array}{l}\text { Completely } \\
\text { not confident }\end{array}$ & $27(8.26)$ & $10(29.41)$ & $3(7.69)$ & $5(13.89)$ & $45(10.32)$ & \\
\hline & Confident & $145(44.34)$ & $15(44.12)$ & $14(35.90)$ & $13(36.11)$ & $187(42.89)$ & \\
\hline & $\begin{array}{l}\text { Not } \\
\text { confident }\end{array}$ & $105(32.11)$ & $6(17.65)$ & $6(15.38)$ & $7(19.44)$ & $124(28.44)$ & \\
\hline
\end{tabular}




\begin{tabular}{|c|c|c|c|c|c|c|c|}
\hline \multirow[t]{2}{*}{ VARIABLES } & \multirow[t]{2}{*}{ CATEGORIES } & \multicolumn{6}{|c|}{ HEALTH CARE GROUPS } \\
\hline & & $\begin{array}{l}\text { DOCTORS } \\
N=327 \\
(75.00 \%)\end{array}$ & $\begin{array}{l}\text { NURSES } \\
\text { N=34 } \\
(7.80 \%)\end{array}$ & $\begin{array}{l}\text { PARAMEDICS } \\
\mathrm{N}=39 \\
(\mathbf{8 . 9 4 \% )}\end{array}$ & $\begin{array}{l}\text { OTHERS } \\
\mathrm{N}=36 \\
(8.26 \%)\end{array}$ & $\begin{array}{l}\text { Total } \\
N=436 \\
(100 \%)\end{array}$ & P-value \\
\hline \multirow{5}{*}{$\begin{array}{l}\text { Vaccination is a } \\
\text { good idea because } \\
\text { I feel less } \\
\text { worried about } \\
\text { getting COVID-19? }\end{array}$} & Agree & $149(45.57)$ & $16(47.06)$ & $18(46.15)$ & $15(41.67)$ & $198(45.41)$ & \multirow[t]{5}{*}{0.12} \\
\hline & Disagree & $11(3.36)$ & $0(0.00)$ & $1(2.56)$ & $1(2.78)$ & $13(2.98)$ & \\
\hline & Neutral & $67(20.49)$ & $11(32.35)$ & $3(7.69)$ & $6(16.67)$ & $87(19.95)$ & \\
\hline & $\begin{array}{l}\text { Strongly } \\
\text { agree }\end{array}$ & $95(29.05)$ & $7(20.59)$ & $15(38.46)$ & $11(30.56)$ & $128(29.36)$ & \\
\hline & $\begin{array}{l}\text { Strongly } \\
\text { disagree }\end{array}$ & $5(1.53)$ & $0(0.00)$ & $2(5.13)$ & $3(8.33)$ & $10(2.29)$ & \\
\hline \multirow{5}{*}{$\begin{array}{l}\text { Vaccination } \\
\text { decreases my } \\
\text { chance of getting } \\
\text { COVID-19 or its } \\
\text { Complications? }\end{array}$} & Agree & 171(52.29) & $12(35.29)$ & $22(56.41)$ & $14(38.89)$ & $219(50.23)$ & \multirow[t]{5}{*}{$<0.001$} \\
\hline & Disagree & $15(4.59)$ & $1(2.94)$ & $1(2.56)$ & $6(16.67)$ & $23(5.28)$ & \\
\hline & Neutral & $57(17.43)$ & $15(44.12)$ & $3(7.69)$ & $5(13.89)$ & $80(18.35)$ & \\
\hline & $\begin{array}{l}\text { Strongly } \\
\text { agree }\end{array}$ & $83(25.38)$ & $6(17.65)$ & $11(28.21)$ & $10(27.78)$ & $110(25.23)$ & \\
\hline & $\begin{array}{l}\text { Strongly } \\
\text { disagree }\end{array}$ & $1(0.31)$ & $0(0.00)$ & $2(5.13)$ & $1(2.78)$ & $4(0.92)$ & \\
\hline \multirow{5}{*}{$\begin{array}{l}\text { I am worried that } \\
\text { the possible side } \\
\text { effects of COVID- } \\
19 \text { vaccination } \\
\text { would interfere } \\
\text { with my routine } \\
\text { activities? }\end{array}$} & Agree & $92(28.13)$ & $18(52.94)$ & $17(43.59)$ & $12(33.33)$ & $139(31.88)$ & \multirow[t]{5}{*}{0.009} \\
\hline & Disagree & $83(25.38)$ & $3(8.82)$ & $7(17.95)$ & $5(13.89)$ & $98(22.48)$ & \\
\hline & Neutral & $117(35.78)$ & $10(29.41)$ & $9(23.08)$ & $10(27.78)$ & $146(33.49)$ & \\
\hline & $\begin{array}{l}\text { Strongly } \\
\text { agree }\end{array}$ & $24(7.34)$ & $3(8.82)$ & $2(5.13)$ & $6(16.67)$ & $35(8.03)$ & \\
\hline & $\begin{array}{l}\text { Strongly } \\
\text { disagree }\end{array}$ & $11(3.36)$ & $0(0.00)$ & $4(10.26)$ & $3(8.33)$ & $18(4.13)$ & \\
\hline \multirow{5}{*}{$\begin{array}{l}\text { I am concerned } \\
\text { about the efficacy } \\
\text { of the COVID-19 } \\
\text { vaccination? }\end{array}$} & Agree & $167(51.07)$ & $14(41.18)$ & $20(51.28)$ & $19(52.78)$ & $220(50.46)$ & \multirow[t]{5}{*}{0.04} \\
\hline & Disagree & $30(9.17)$ & $3(8.82)$ & $0(0.00)$ & $1(2.78)$ & $34(7.80)$ & \\
\hline & Neutral & $70(21.41)$ & $15(44.12)$ & $10(25.64)$ & $7(19.44)$ & $102(23.39)$ & \\
\hline & $\begin{array}{l}\text { Strongly } \\
\text { agree }\end{array}$ & $56(17.13)$ & $2(5.88)$ & $7(17.95)$ & $7(19.44)$ & $72(16.51)$ & \\
\hline & $\begin{array}{l}\text { Strongly } \\
\text { disagree }\end{array}$ & $4(1.22)$ & $0(0.00)$ & $2(5.13)$ & $2(5.56)$ & $8(1.83)$ & \\
\hline \multirow{5}{*}{$\begin{array}{l}\text { I am concerned } \\
\text { about the safety of } \\
\text { the COVID-19 } \\
\text { vaccination? }\end{array}$} & Agree & $150(45.87)$ & $18(52.94)$ & $17(43.59)$ & $19(52.78)$ & 204(46.79) & \multirow[t]{5}{*}{0.10} \\
\hline & Disagree & $37(11.31)$ & $2(5.88)$ & $4(10.26)$ & $1(2.78)$ & $44(10.09)$ & \\
\hline & Neutral & $69(21.10)$ & $12(35.29)$ & $6(15.38)$ & $4(11.11)$ & $91(20.87)$ & \\
\hline & $\begin{array}{l}\text { Strongly } \\
\text { agree }\end{array}$ & $66(20.18)$ & $2(5.88)$ & $10(25.64)$ & $11(30.56)$ & $89(20.41)$ & \\
\hline & $\begin{array}{l}\text { Strongly } \\
\text { disagree }\end{array}$ & $5(1.53)$ & $0(0.00)$ & $2(5.13)$ & $1(2.78)$ & $8(1.83)$ & \\
\hline \multirow{5}{*}{$\begin{array}{l}\text { I am concerned of } \\
\text { the faulty/fake } \\
\text { COVID-19 } \\
\text { vaccination? }\end{array}$} & Agree & $126(38.53)$ & $4(11.76)$ & $12(30.77)$ & $15(41.67)$ & $157(36.01)$ & \multirow[t]{5}{*}{$<0.001$} \\
\hline & Disagree & $61(18.65)$ & $12(35.29)$ & $13(33.33)$ & $6(16.67)$ & $92(21.10)$ & \\
\hline & Neutral & $66(20.18)$ & $15(44.12)$ & $5(12.82)$ & $1(2.78)$ & $87(19.95)$ & \\
\hline & $\begin{array}{l}\text { Strongly } \\
\text { agree }\end{array}$ & 64(19.57) & $2(5.88)$ & $6(15.38)$ & $9(25.00)$ & $81(18.58)$ & \\
\hline & $\begin{array}{l}\text { Strongly } \\
\text { disagree }\end{array}$ & $10(3.06)$ & $1(2.94)$ & $3(7.69)$ & $5(13.89)$ & $19(4.36)$ & \\
\hline
\end{tabular}


02 c: COVID-19 vaccine acceptance, barriers and facilitators amongst health care workers of Pakistan

\begin{tabular}{|c|c|c|c|c|c|c|c|}
\hline \multirow[t]{2}{*}{ BLES } & \multirow[t]{2}{*}{ CATEGORIES } & \multicolumn{6}{|c|}{ HEALTH CARE GROUPS } \\
\hline & & $\begin{array}{l}\text { DOCTORS } \\
N=327 \\
(75.00 \%)\end{array}$ & $\begin{array}{l}\text { NURSES } \\
N=34 \\
(7.80 \%)\end{array}$ & $\begin{array}{l}\text { PARAMEDICS } \\
\mathrm{N}=39 \\
(8.94 \%)\end{array}$ & $\begin{array}{l}\text { OTHERS } \\
\mathrm{N}=36 \\
(8.26 \%)\end{array}$ & $\begin{array}{l}\text { Total } \\
N=436 \\
(100 \%)\end{array}$ & P-value \\
\hline \multirow{5}{*}{$\begin{array}{l}\text { oncerned about } \\
\text { Cold Chain } \\
\text { nance of } \\
\text {-19 vaccine? }\end{array}$} & Agree & $148(45.26)$ & $17(50.00)$ & $15(38.46)$ & $14(38.89)$ & $194(44.50)$ & \multirow[t]{5}{*}{0.12} \\
\hline & Disagree & $21(6.42)$ & $2(5.88)$ & $2(5.13)$ & $2(5.56)$ & $27(6.19)$ & \\
\hline & Neutral & $58(17.74)$ & $9(26.47)$ & $10(25.64)$ & $9(25.00)$ & $86(19.72)$ & \\
\hline & $\begin{array}{l}\text { Strongly } \\
\text { agree }\end{array}$ & $99(30.28)$ & $6(17.65)$ & $10(25.64)$ & $11(30.56)$ & $126(28.90)$ & \\
\hline & $\begin{array}{l}\text { Strongly } \\
\text { disagree }\end{array}$ & $1(0.31)$ & $0(0.00)$ & $2(5.13)$ & $0(0.00)$ & $3(0.69)$ & \\
\hline \multirow{5}{*}{$\begin{array}{l}\text { oncerned about } \\
\text { ssibility of } \\
\text { )-19 vaccine on } \\
\text { us grounds? }\end{array}$} & Agree & $56(17.13)$ & $12(35.29)$ & $10(25.64)$ & $10(27.78)$ & $88(20.18)$ & \multirow[t]{5}{*}{0.005} \\
\hline & Disagree & $121(37.00)$ & $9(26.47)$ & $7(17.95)$ & $11(30.56)$ & $148(33.94)$ & \\
\hline & Neutral & $83(25.38)$ & $12(35.29)$ & $12(30.77)$ & $8(22.22)$ & 115(26.38) & \\
\hline & $\begin{array}{l}\text { Strongly } \\
\text { agree }\end{array}$ & $24(7.34)$ & $1(2.94)$ & $8(20.51)$ & $4(11.11)$ & $37(8.49)$ & \\
\hline & $\begin{array}{l}\text { Strongly } \\
\text { disagree }\end{array}$ & $43(13.15)$ & $0(0.00)$ & $2(5.13)$ & $3(8.33)$ & $48(11.01)$ & \\
\hline \multirow{5}{*}{$\begin{array}{l}\text { might be } \\
1 \text { motives } \\
1 \text { eagerness of } \\
\text { ities to } \\
\text { ister/provide } \\
\text { e free for all } \\
\text { workers? }\end{array}$} & Agree & $47(14.37)$ & $16(47.06)$ & $9(23.08)$ & $11(30.56)$ & $83(19.04)$ & \multirow[t]{5}{*}{$<0.001$} \\
\hline & Disagree & $111(33.94)$ & $4(11.76)$ & $9(23.08)$ & $8(22.22)$ & $132(30.28)$ & \\
\hline & Neutral & $83(25.38)$ & $8(23.53)$ & $10(25.64)$ & $7(19.44)$ & $108(24.77)$ & \\
\hline & $\begin{array}{l}\text { Strongly } \\
\text { agree }\end{array}$ & $26(7.95)$ & $6(17.65)$ & $8(20.51)$ & $5(13.89)$ & $45(10.32)$ & \\
\hline & $\begin{array}{l}\text { Strongly } \\
\text { disagree }\end{array}$ & $60(18.35)$ & $0(0.00)$ & $3(7.69)$ & $5(13.89)$ & $68(15.60)$ & \\
\hline
\end{tabular}




\begin{tabular}{|c|c|c|c|c|c|c|c|}
\hline \multirow[t]{2}{*}{ VARIABLE } & \multirow[t]{2}{*}{ CATEGORIES } & \multicolumn{6}{|c|}{ HEALTH CARE GROUPS } \\
\hline & & $\begin{array}{l}\text { DOCTORS } \\
\text { N=242 } \\
(72.24 \%) \\
\end{array}$ & $\begin{array}{l}\text { NURSES } \\
\mathrm{N}=29 \\
(8.66 \%)\end{array}$ & $\begin{array}{l}\text { PARAMEDICS } \\
\mathrm{N}=37 \\
(11.04 \%)\end{array}$ & \begin{tabular}{|l|} 
OTHERS \\
$\mathrm{N}=27$ \\
$(8.06 \%)$ \\
\end{tabular} & $\begin{array}{l}\text { Total } \\
N=335 \\
(100 \%) \\
\end{array}$ & P-value \\
\hline \multirow[t]{2}{*}{ Gender } & Female & $41(16.94)$ & $15(51.72)$ & $3(8.11)$ & $3(11.11)$ & $62(18.51)$ & \multirow[t]{2}{*}{$<0.001$} \\
\hline & Male & $201(83.06)$ & $14(48.28)$ & $34(91.89)$ & $24(88.89)$ & $273(81.49)$ & \\
\hline \multirow{3}{*}{$\begin{array}{l}\text { Category of } \\
\text { age }\end{array}$} & $19-39$ & $166(68.60)$ & $28(96.55)$ & $28(75.68)$ & $25(92.59)$ & $247(73.73)$ & \multirow[t]{3}{*}{0.01} \\
\hline & $40-60$ & $68(28.10)$ & $1(3.45)$ & $8(21.62)$ & $2(7.41)$ & $79(23.58)$ & \\
\hline & $61-81$ & $8(3.31)$ & $0(0.00)$ & $1(2.70)$ & $0(0.00)$ & $9(2.69)$ & \\
\hline \multirow{3}{*}{$\begin{array}{l}\text { Category of } \\
\text { work } \\
\text { experience in } \\
\text { years }\end{array}$} & $0-5$ Years & $100(41.32)$ & $19(65.52)$ & $21(56.76)$ & $15(55.56)$ & $155(46.27)$ & \multirow[t]{3}{*}{0.006} \\
\hline & 6-10 Years & $62(25.62)$ & $8(27.59)$ & $4(10.81)$ & $9(33.33)$ & $83(24.78)$ & \\
\hline & Above 10 Years & $80(33.06)$ & $2(6.90)$ & $12(32.43)$ & $3(11.11)$ & $97(28.96)$ & \\
\hline \multirow{6}{*}{$\begin{array}{l}\text { Working at } \\
\text { which health } \\
\text { care level? }\end{array}$} & $\begin{array}{l}\text { COVID-19 } \\
\text { Facility/Hospitals }\end{array}$ & $3(1.24)$ & $0(0.00)$ & $6(16.22)$ & $1(3.70)$ & $10(2.99)$ & \multirow[t]{6}{*}{$<0.001$} \\
\hline & $\begin{array}{l}\text { Emergency } \\
\text { Operation } \\
\text { Center(National, } \\
\text { provincials and } \\
\text { districts) }\end{array}$ & $26(10.74)$ & $0(0.00)$ & $1(2.70)$ & $2(7.41)$ & $29(8.66)$ & \\
\hline & $\begin{array}{l}\text { Primary Health } \\
\text { Care Facility }\end{array}$ & $29(11.98)$ & $0(0.00)$ & $9(24.32)$ & $0(0.00)$ & $38(11.34)$ & \\
\hline & $\begin{array}{l}\text { Secondary Health } \\
\text { Care Facility }\end{array}$ & $60(24.79)$ & $11(37.93)$ & $7(18.92)$ & $3(11.11)$ & $81(24.18)$ & \\
\hline & $\begin{array}{l}\text { Tertiary Health } \\
\text { Care Facility }\end{array}$ & $106(43.80)$ & $16(55.17)$ & $9(24.32)$ & $7(25.93)$ & $138(41.19)$ & \\
\hline & Other & $18(7.44)$ & $2(6.90)$ & $5(13.51)$ & $14(51.85)$ & $39(11.64)$ & \\
\hline \multirow{3}{*}{$\begin{array}{l}\text { Are } \quad \text { you } \\
\text { working at } \\
\text { facility/ward } \\
\text { dedicated for } \\
\text { COVID-19 } \\
\text { patients? }\end{array}$} & No & $109(45.04)$ & $10(34.48)$ & $15(40.54)$ & $15(55.56)$ & $149(44.48)$ & \multirow[t]{3}{*}{0.03} \\
\hline & Yes & $87(35.95)$ & $13(44.83)$ & $20(54.05)$ & $4(14.81)$ & $124(37.01)$ & \\
\hline & Both & $46(19.01)$ & $6(20.69)$ & $2(5.41)$ & $8(29.63)$ & $62(18.51)$ & \\
\hline
\end{tabular}




\begin{tabular}{|c|c|c|c|c|c|c|c|}
\hline \multirow[t]{2}{*}{ VARIABLES } & \multirow[t]{2}{*}{ CATEGORIES } & \multicolumn{6}{|c|}{ HEALTH CARE GROUPS } \\
\hline & & $\begin{array}{l}\text { DOCTORS } \\
N=242 \\
\text { (72.24\%) }\end{array}$ & $\begin{array}{l}\text { NURSES } \\
\text { N=29 } \\
(8.66 \%)\end{array}$ & $\begin{array}{l}\text { PARAMEDICS } \\
\text { N=37 } \\
(11.04 \%)\end{array}$ & $\begin{array}{l}\text { OTHERS } \\
\mathrm{N}=27 \\
(8.06 \%)\end{array}$ & $\begin{array}{l}\text { Total } \\
\mathrm{N}=335 \\
(100 \%)\end{array}$ & P-value \\
\hline \multirow{2}{*}{$\begin{array}{l}\text { Have you ever } \\
\text { tested } \\
\text { Positive for } \\
\text { COVID-19? }\end{array}$} & No & $166(68.60)$ & $19(65.52)$ & $28(75.68)$ & $20(74.07)$ & $233(69.55)$ & \multirow[t]{2}{*}{0.74} \\
\hline & Yes & $76(31.40)$ & $10(34.48)$ & $9(24.32)$ & $7(25.93)$ & $102(30.45)$ & \\
\hline \multirow{2}{*}{$\begin{array}{l}\text { Do you have an } \\
\text { existing chronic } \\
\text { disease such as } \\
\text { cardiovascular } \\
\text { disease, diabetes, } \\
\text { Chronic } \\
\text { Respiratory tract } \\
\text { disease? }\end{array}$} & No & $203(83.88)$ & 28(96.55) & $34(91.89)$ & $24(88.89)$ & $289(86.27)$ & \multirow[t]{2}{*}{0.18} \\
\hline & Yes & $39(16.12)$ & $1(3.45)$ & $3(8.11)$ & $3(11.11)$ & $46(13.73)$ & \\
\hline \multirow{3}{*}{$\begin{array}{ll}\text { You believe } & \text { you } \\
\text { have } & \\
\text { Adequate } & \\
\text { information } & \\
\text { regarding } & \text { the } \\
\text { Covid19 } & \\
\text { Vaccine? } & \end{array}$} & I am not sure & $82(33.88)$ & $12(41.38)$ & $14(37.84)$ & $10(37.04)$ & $118(35.22)$ & \multirow[t]{3}{*}{0.40} \\
\hline & No & $53(21.90)$ & $8(27.59)$ & $3(8.11)$ & $5(18.52)$ & $69(20.60)$ & \\
\hline & Yes & $107(44.21)$ & $9(31.03)$ & $20(54.05)$ & $12(44.44)$ & $148(44.18)$ & \\
\hline \multirow{4}{*}{$\begin{array}{l}\text { If vaccine against } \\
\text { COVID-19 infection } \\
\text { is available would } \\
\text { You take it? }\end{array}$} & I am not sure & 13(5.37) & $3(10.34)$ & $2(5.41)$ & $1(3.70)$ & $19(5.67)$ & \multirow[t]{4}{*}{0.35} \\
\hline & $\begin{array}{l}\text { I will wait } \\
\text { and see }\end{array}$ & $75(30.99)$ & $12(41.38)$ & $11(29.73)$ & $12(44.44)$ & $110(32.84)$ & \\
\hline & No & $8(3.31)$ & $1(3.45)$ & $1(2.70)$ & $3(11.11)$ & $13(3.88)$ & \\
\hline & $\overline{\text { Yes }}$ & $146(60.33)$ & $13(44.83)$ & $23(62.16)$ & $11(40.74)$ & $193(57.61)$ & \\
\hline \multirow{4}{*}{$\begin{array}{l}\text { Rate } \\
\text { confidence in } \\
\text { using UK,USA } \\
\text { manufactured } \\
\text { (imported) } \\
\text { COVID-19 vaccine? }\end{array}$} & $\begin{array}{l}\text { Completely } \\
\text { confident }\end{array}$ & $47(19.42)$ & $6(20.69)$ & $11(29.73)$ & $7(25.93)$ & $71(21.19)$ & \multirow[t]{4}{*}{0.002} \\
\hline & $\begin{array}{l}\text { Completely } \\
\text { not confident }\end{array}$ & $20(8.26)$ & $10(34.48)$ & $4(10.81)$ & $5(18.52)$ & $39(11.64)$ & \\
\hline & Confident & $131(54.13)$ & $8(27.59)$ & $14(37.84)$ & $8(29.63)$ & $161(48.06)$ & \\
\hline & $\begin{array}{l}\text { Not } \\
\text { confident }\end{array}$ & $44(18.18)$ & $5(17.24)$ & $8(21.62)$ & $7(25.93)$ & $64(19.10)$ & \\
\hline \multirow{4}{*}{$\begin{array}{l}\text { Rate your } \\
\text { confidence in } \\
\text { Using China } \\
\text { manufactured } \\
\text { COVID-19 vaccine? }\end{array}$} & $\begin{array}{l}\text { Completely } \\
\text { confident }\end{array}$ & $39(16.12)$ & $3(10.34)$ & $15(40.54)$ & $7(25.93)$ & $64(19.10)$ & \multirow[t]{4}{*}{$<0.001$} \\
\hline & $\begin{array}{l}\text { Completely } \\
\text { not confident }\end{array}$ & $21(8.68)$ & $9(31.03)$ & $3(8.11)$ & $5(18.52)$ & $38(11.34)$ & \\
\hline & Confident & $107(44.21)$ & $13(44.83)$ & $14(37.84)$ & $8(29.63)$ & $142(42.39)$ & \\
\hline & $\begin{array}{l}\text { Not } \\
\text { confident }\end{array}$ & $75(30.99)$ & $4(13.79)$ & $5(13.51)$ & $7(25.93)$ & $91(27.16)$ & \\
\hline
\end{tabular}


Table 03( c ) COVID-19 Vaccine Acceptance, barriers and facilitators amongst health care workers of Province of Khyber Pakhtunkhwa Pakistan

\begin{tabular}{|c|c|c|c|c|c|c|c|}
\hline \multirow[t]{2}{*}{ VARIABLES } & \multirow[t]{2}{*}{ CATEGORIES } & \multicolumn{6}{|c|}{ HEALTH CARE GROUPS } \\
\hline & & $\begin{array}{l}\text { DOCTORS } \\
\text { N=242 } \\
(72.24 \%) \\
\end{array}$ & $\begin{array}{l}\text { NURSES } \\
\text { N=29 } \\
(8.66 \%) \\
\end{array}$ & $\begin{array}{l}\text { PARAMEDICS } \\
\mathrm{N}=37 \\
(11.04 \%)\end{array}$ & $\begin{array}{l}\text { OTHERS } \\
\mathrm{N}=27 \\
(8.06 \%) \\
\end{array}$ & $\begin{array}{l}\text { Total } \\
N=335 \\
(100 \%) \\
\end{array}$ & P-value \\
\hline \multirow{5}{*}{$\begin{array}{l}\text { Vaccination is a } \\
\text { good idea because } \\
\text { I feel less } \\
\text { worried about } \\
\text { getting COVID-19? }\end{array}$} & Agree & $112(46.28)$ & $15(51.72)$ & $17(45.95)$ & $10(37.04)$ & $154(45.97)$ & \multirow[t]{5}{*}{0.30} \\
\hline & Disagree & $6(2.48)$ & $0(0.00)$ & $1(2.70)$ & $1(3.70)$ & $8(2.39)$ & \\
\hline & Neutral & $49(20.25)$ & $7(24.14)$ & $3(8.11)$ & $5(18.52)$ & $64(19.10)$ & \\
\hline & $\begin{array}{l}\text { Strongly } \\
\text { agree }\end{array}$ & $70(28.93)$ & $7(24.14)$ & $14(37.84)$ & $8(29.63)$ & $99(29.55)$ & \\
\hline & $\begin{array}{l}\text { Strongly } \\
\text { disagree }\end{array}$ & $5(2.07)$ & $0(0.00)$ & $2(5.41)$ & $3(11.11)$ & $10(2.99)$ & \\
\hline \multirow{5}{*}{$\begin{array}{l}\text { Vaccination } \\
\text { decreases my } \\
\text { chance of getting } \\
\text { COVID-19 or its } \\
\text { Complications? }\end{array}$} & Agree & 123(50.83) & $12(41.38)$ & $20(54.05)$ & $10(37.04)$ & $165(49.25)$ & \multirow[t]{5}{*}{0.002} \\
\hline & Disagree & $12(4.96)$ & $1(3.45)$ & $1(2.70)$ & $6(22.22)$ & $20(5.97)$ & \\
\hline & Neutral & $42(17.36)$ & $10(34.48)$ & $3(8.11)$ & $3(11.11)$ & $58(17.31)$ & \\
\hline & $\begin{array}{l}\text { Strongly } \\
\text { agree }\end{array}$ & $64(26.45)$ & $6(20.69)$ & $11(29.73)$ & $7(25.93)$ & $88(26.27)$ & \\
\hline & $\begin{array}{l}\text { Strongly } \\
\text { disagree }\end{array}$ & $1(0.41)$ & $0(0.00)$ & $2(5.41)$ & $1(3.70)$ & $4(1.19)$ & \\
\hline \multirow{5}{*}{$\begin{array}{l}\text { I am worried that } \\
\text { the possible side } \\
\text { effects of COVID- } \\
19 \text { vaccination } \\
\text { would interfere } \\
\text { with my routine } \\
\text { activities? }\end{array}$} & Agree & $66(27.27)$ & $16(55.17)$ & $16(43.24)$ & $9(33.33)$ & $107(31.94)$ & \multirow[t]{5}{*}{0.07} \\
\hline & Disagree & $58(23.97)$ & $3(10.34)$ & $7(18.92)$ & $3(11.11)$ & $71(21.19)$ & \\
\hline & Neutral & $90(37.19)$ & $7(24.14)$ & $9(24.32)$ & $9(33.33)$ & $115(34.33)$ & \\
\hline & $\begin{array}{l}\text { Strongly } \\
\text { agree }\end{array}$ & $18(7.44)$ & $3(10.34)$ & $2(5.41)$ & $3(11.11)$ & $26(7.76)$ & \\
\hline & $\begin{array}{l}\text { Strongly } \\
\text { disagree }\end{array}$ & $10(4.13)$ & $0(0.00)$ & $3(8.11)$ & $3(11.11)$ & $16(4.78)$ & \\
\hline \multirow{5}{*}{$\begin{array}{l}\text { I am concerned } \\
\text { about the efficacy } \\
\text { of the COVID-19 } \\
\text { vaccination? }\end{array}$} & Agree & $128(52.89)$ & $10(34.48)$ & $20(54.05)$ & $14(51.85)$ & $172(51.34)$ & \multirow[t]{5}{*}{0.02} \\
\hline & Disagree & $23(9.50)$ & $3(10.34)$ & $0(0.00)$ & $1(3.70)$ & $27(8.06)$ & \\
\hline & Neutral & $48(19.83)$ & $14(48.28)$ & $9(24.32)$ & $6(22.22)$ & $77(22.99)$ & \\
\hline & $\begin{array}{l}\text { Strongly } \\
\text { agree }\end{array}$ & $40(16.53)$ & $2(6.90)$ & $6(16.22)$ & $4(14.81)$ & $52(15.52)$ & \\
\hline & $\begin{array}{l}\text { Strongly } \\
\text { disagree }\end{array}$ & $3(1.24)$ & $0(0.00)$ & $2(5.41)$ & $2(7.41)$ & $7(2.09)$ & \\
\hline \multirow{5}{*}{$\begin{array}{l}\text { I am concerned } \\
\text { about the safety of } \\
\text { the COVID-19 } \\
\text { vaccination? }\end{array}$} & Agree & $113(46.69)$ & $14(48.28)$ & $17(45.95)$ & $14(51.85)$ & $158(47.16)$ & \multirow[t]{5}{*}{0.20} \\
\hline & Disagree & $24(9.92)$ & $2(6.90)$ & $4(10.81)$ & $1(3.70)$ & $31(9.25)$ & \\
\hline & Neutral & $52(21.49)$ & $11(37.93)$ & $5(13.51)$ & $3(11.11)$ & $71(21.19)$ & \\
\hline & $\begin{array}{l}\text { Strongly } \\
\text { agree }\end{array}$ & $50(20.66)$ & $2(6.90)$ & $9(24.32)$ & $8(29.63)$ & $69(20.60)$ & \\
\hline & $\begin{array}{l}\text { Strongly } \\
\text { disagree }\end{array}$ & $3(1.24)$ & $0(0.00)$ & $2(5.41)$ & $1(3.70)$ & $6(1.79)$ & \\
\hline \multirow{5}{*}{$\begin{array}{l}\text { I am concerned of } \\
\text { the faulty/fake } \\
\text { COVID-19 } \\
\text { vaccination? }\end{array}$} & Agree & $88(36.36)$ & $2(6.90)$ & $11(29.73)$ & $11(40.74)$ & $112(33.43)$ & \multirow[t]{5}{*}{$<0.001$} \\
\hline & Disagree & $47(19.42)$ & $12(41.38)$ & $13(35.14)$ & $4(14.81)$ & $76(22.69)$ & \\
\hline & Neutral & $50(20.66)$ & $12(41.38)$ & $5(13.51)$ & $1(3.70)$ & $68(20.30)$ & \\
\hline & $\begin{array}{l}\text { Strongly } \\
\text { agree }\end{array}$ & $50(20.66)$ & $2(6.90)$ & $5(13.51)$ & $6(22.22)$ & $63(18.81)$ & \\
\hline & $\begin{array}{l}\text { Strongly } \\
\text { disagree }\end{array}$ & 7(2.89) & $1(3.45)$ & $3(8.11)$ & $5(18.52)$ & $16(4.78)$ & \\
\hline
\end{tabular}




\begin{tabular}{|c|c|c|c|c|c|c|c|}
\hline \multicolumn{3}{|c|}{$\begin{array}{l}\text { Table 03(d) COVID-19 Vaccine Acceptance, } \\
\text { of Province of Khyber Pakhtunkhwa Pakistan }\end{array}$} & & \multicolumn{3}{|c|}{ amongst health care workers } \\
\hline \multirow[t]{2}{*}{ VARIABLES } & \multirow[t]{2}{*}{ CATEGORIES } & \multicolumn{6}{|c|}{ HEALTH CARE GROUPS } \\
\hline & & $\begin{array}{l}\text { DOCTORS } \\
N=242 \\
\text { (72.24\%) }\end{array}$ & $\begin{array}{l}\text { NURSES } \\
\mathrm{N}=29 \\
\text { (8.66 \%) }\end{array}$ & $\begin{array}{l}\text { PARAMEDICS } \\
\mathrm{N}=37 \\
(11.04 \%)\end{array}$ & $\begin{array}{l}\text { OTHERS } \\
\mathrm{N}=27 \\
(8.06 \%)\end{array}$ & $\begin{array}{l}\text { Total } \\
\mathrm{N}=335 \\
(100 \%)\end{array}$ & $\begin{array}{l}\text { P- } \\
\text { value }\end{array}$ \\
\hline \multirow{5}{*}{$\begin{array}{l}\text { I am concerned } \\
\text { about the Cold } \\
\text { Chain } \\
\text { maintenance of } \\
\text { COVID-19 vaccine? }\end{array}$} & Agree & $109(45.04)$ & $13(44.83)$ & $14(37.84)$ & $12(44.44)$ & $148(44.18)$ & \multirow[t]{5}{*}{0.32} \\
\hline & Disagree & $14(5.79)$ & $2(6.90)$ & $2(5.41)$ & $2(7.41)$ & $20(5.97)$ & \\
\hline & Neutral & $45(18.60)$ & $8(27.59)$ & $10(27.03)$ & $7(25.93)$ & $70(20.90)$ & \\
\hline & $\begin{array}{l}\text { Strongly } \\
\text { agree }\end{array}$ & $73(30.17)$ & $6(20.69)$ & $9(24.32)$ & $6(22.22)$ & $94(28.06)$ & \\
\hline & $\begin{array}{l}\text { Strongly } \\
\text { disagree }\end{array}$ & $1(0.41)$ & $0(0.00)$ & $2(5.41)$ & $0(0.00)$ & $3(0.90)$ & \\
\hline \multirow{6}{*}{$\begin{array}{l}\text { I am concerned } \\
\text { about } \\
\text { permissibility of } \\
\text { COVI-19 vaccine } \\
\text { on religious } \\
\text { grounds? }\end{array}$} & Agree & $42(17.36)$ & $10(34.48)$ & $10(27.03)$ & $7(25.93)$ & $69(20.60)$ & \multirow[t]{5}{*}{0.03} \\
\hline & Disagree & $84(34.71)$ & $9(31.03)$ & $6(16.22)$ & $8(29.63)$ & $107(31.94)$ & \\
\hline & Neutral & $70(28.93)$ & $9(31.03)$ & $11(29.73)$ & $7(25.93)$ & $97(28.96)$ & \\
\hline & $\begin{array}{l}\text { Strongly } \\
\text { agree }\end{array}$ & $17(7.02)$ & $1(3.45)$ & $8(21.62)$ & $2(7.41)$ & $28(8.36)$ & \\
\hline & \multirow[t]{2}{*}{$\begin{array}{l}\text { Strongly } \\
\text { disagree }\end{array}$} & \multirow[t]{2}{*}{$29(11.98)$} & \multirow[t]{2}{*}{$0(0.00)$} & \multirow[t]{2}{*}{$2(5.41)$} & \multirow[t]{2}{*}{$3(11.11)$} & \multirow[t]{2}{*}{$34(10.15)$} & \\
\hline & & & & & & & \multirow[t]{6}{*}{0.001} \\
\hline \multirow{5}{*}{$\begin{array}{l}\text { There might be } \\
\text { hidden motives } \\
\text { behind eagerness } \\
\text { of authorities to } \\
\text { administer/provide } \\
\text { vaccine free for all } \\
\text { health workers? }\end{array}$} & Agree & $40(16.53)$ & $14(48.28)$ & $9(24.32)$ & $8(29.63)$ & $71(21.19)$ & \\
\hline & Disagree & $81(33.47)$ & $3(10.34)$ & $7(18.92)$ & $6(22.22)$ & $97(28.96)$ & \\
\hline & Neutral & $63(26.03)$ & $7(24.14)$ & $10(27.03)$ & $6(22.22)$ & $86(25.67)$ & \\
\hline & $\begin{array}{l}\text { Strongly } \\
\text { agree }\end{array}$ & $20(8.26)$ & $5(17.24)$ & $8(21.62)$ & $2(7.41)$ & $35(10.45)$ & \\
\hline & $\begin{array}{l}\text { Strongly } \\
\text { disagree }\end{array}$ & $38(15.70)$ & $0(0.00)$ & $3(8.11)$ & $5(18.52)$ & $46(13.73)$ & \\
\hline
\end{tabular}

